\title{
Reflets
}

Revue ontaroise d'intervention sociale et communautaire

\section{L'approche institutionnelle répond-t-elle aux besoins de sa clientèle?}

\section{Danielle Perron-Roach}

Volume 4, numéro 2, automne 1998

Personnes vivant avec une incapacité

URI : https://id.erudit.org/iderudit/026220ar

DOI : https://doi.org/10.7202/026220ar

Aller au sommaire du numéro

Éditeur(s)

Reflets : Revue ontaroise d'intervention sociale et communautaire

ISSN

1203-4576 (imprimé)

1712-8498 (numérique)

Découvrir la revue

Citer cet article

Perron-Roach, D. (1998). L’approche institutionnelle répond-t-elle aux besoins de sa clientèle? Reflets, 4(2), 106-114. https://doi.org/10.7202/026220ar

Tous droits réservés (C) Reflets : Revue ontaroise d'intervention sociale et communautaire, 1998
Ce document est protégé par la loi sur le droit d'auteur. L'utilisation des services d'Érudit (y compris la reproduction) est assujettie à sa politique d'utilisation que vous pouvez consulter en ligne.

https://apropos.erudit.org/fr/usagers/politique-dutilisation/ 


\title{
L'approche institutionnelle répond-t-elle aux besoins de sa clientèle?
}

\author{
Danielle Perron-Roach \\ Travailleuse sociale, Hôpital Montfort, Ottawa
}

\section{Introduction}

Il y a quatre ans, j'ai terminé une maîtrise à l'École de service social de l'université d'Ottawa. Depuis, j'occupe le poste de travailleuse sociale dans un hôpital communautaire qui dessert une population francophone dans une région majoritairement anglophone. J'ai travaillé dans tous les départements de l'hôpital (santé mentale, salle d'urgence, unités d'orthopédie, de chirurgie et d'obstétrique). J'agis également comme conseillère en matière de droits, tels que définis par l'Acte sur la santé mentale et la Loi sur le consentement. Les clients avec lesquels je travaille font face à la fois à des problèmes de santé et des problèmes sociaux, les deux étant souvent interdépendants. Par exemple, une femme âgée de 82 ans peut être incapable de retourner vivre seule dans sa maison à la suite d'une fracture de la hanche. Dans un autre cas, une perte d'emploi ou un divorce, voire les deux, peut provoquer une dépression majeure chez un individu.

Quelques mois sur le marché du travail m'ont suffi pour voir à quel point le marché du travail présentait une réalité bien différente de celle présentée par le monde académique. Cela dit, j'ai appris, à l'université, à remettre en question, à réfléchir et à 
analyser certaines problématiques. L'exercice académique qui m'a permis de découvrir et de développer les valeurs professionnelles qui orientent aujourd'hui mes interventions, a été la recherche et la rédaction de mon mémoire de maitrise, intitulé : «J'veux juste faire ma vie! Les pratiques de débrouillardise des personnes ayant un handicap physique». Ma recherche explorait alors les stratégies, mises en œuvre par les répondants, pour gérer les contraintes imposées par la société et leurs pratiques de débrouillardise propres à favoriser et à maintenir leur autonomie. En effet, les personnes ayant un handicap physique doivent affronter plusieurs obstacles à la fois relationnels et systémiques. À cet égard, l'approche institutionnelle de type unilatéral contribue, par sa nature même, à l'exclusion des personnes marginalisées de notre société.

Bien que le mémoire ne porte que sur les personnes ayant un handicap physique, les données de cette étude peuvent être représentatives du discours tenu par plusieurs groupes marginalisés de notre société. En effet, le désir de développer et de maintenir son autonomie est un besoin qui préoccupe énormément les clients auprès desquels je dois intervenir régulièrement.

Je présenterai d'abord un bref résumé de mon mémoire de maitrise qui servira à orienter mon propos. Mon mémoire critique énormément l'approche institutionnelle, à laquelle je participe dorénavant. Je discuterai ensuite d'un cas qui révèle certains obstacles imposés par le système institutionnel dans notre lutte pour respecter l'autonomie des clients et quelques stratégies utilisées pour tenter de les contourner.

\section{Résumé du mémoire}

\section{La problématique}

La recherche cerne la problématique des handicaps physiques dans un contexte de désinstitutionnalisation. Pour ce faire, l'étude s'appuie sur le discours de cinq personnes vivant avec différents types de handicaps physiques. La démarche suivie cherchait à saisir 
les personnes participantes comme des acteurs, capables de prendre en charge leur vécu et non, comme des sujets passifs, victimes de leur «infirmité». Le handicap n'est plus alors perçu comme une déficience, mais comme un problème découlant d'une variété de contraintes physiques, sociales et économiques qui marquent l'existence quotidienne des individus.

La recherche exploratoire effectuée est structurée autour de deux axes étroitement liés. Il s'agit d'abord d'explorer le sens que les personnes répondantes donnent à l'expérience de vivre avec leur handicap physique dans notre société et, ensuite, d'identifier les stratégies qu'elles déploient pour survivre aux situations qu'elles ont à affronter quotidiennement. Plus particulièrement, nous désirions connaître les réseaux sur lesquels elles peuvent compter pour développer et maintenir leur autonomie.

L'autonomie est largement déterminée par les réseaux de soutien, dans la mesure où elle se développe au cours de relations d'échange avec l'environnement. Cette étude exploratoire perçoit donc l'autonomie en terme de liens aux autres, contrairement aux définitions traditionnelles qui la définissent comme la capacité de fonctionner par soi-même. Surmontant les divers obstacles, les personnes ayant des handicaps physiques développent des stratégies afin de maintenir leur autonomie. Les répondants ont été capables de mobiliser les ressources et de combattre les préjugés et les barrières qui empêchent leur intégration dans notre société.

\section{Les résultats}

Les témoignages des personnes répondantes démontrent clairement leur désir de vouloir prendre en main leur vie en s'attribuant la liberté de faire des choix et de prendre leurs propres décisions. Or, les préjugés sociaux envers les personnes ayant des handicaps physiques les posent comme des êtres passifs, incapables de se prendre en charge. Cette attitude domine l'intervention institutionnelle, caractérisée par un processus unilatéral qui cherche à exclure les aidés des relations d'échange avec les autres et les empêche de s'intégrer à leur communauté. Maintenir la réciprocité avec les gens de leur entourage est donc la stratégie la plus 
couramment utilisée par les personnes répondantes pour maintenir leur autonomie. Il s'agit d'un effort volontaire pour penser la réciprocité à tous les niveaux, y compris avec les intervenants. Elle leur permet de participer activement au rapport d'échange entre l'aidant et l'aidé. L'intervention professionnelle a tendance à s'arroger le traitement des personnes ayant des handicaps, en présumant connaître ce qui leur convient le mieux pour assurer leur bien-être. Contrairement aux principes de ce type d'aide, les répondants ont démontré qu'ils sont des personnes actives, capables d'assumer la responsabilité de leur propre vie, en développant quotidiennement des stratégies pour surmonter les problèmes liés aux handicaps. L'autonomie et l'amitié sont les valeurs qui dominent leurs actions.

\section{Contraintes de l'intervention en milieu institutionnel : un cas}

\section{Ma tâche}

En tant que travailleuse sociale en milieu hospitalier, une de mes premières tâches est d'assurer la continuité des soins des usagers suite à leur hospitalisation. En milieu hospitalier, le principe fondamental qui domine est que le bien de la société a préséance sur les besoins individuels. L'hôpital a comme responsabilité de répondre aux besoins de ceux nécessitant des soins aigus et donc, le système de santé s'attend à ce que la communauté assume la responsabilité de la continuité des soins. Dans mes fonctions, je dois faciliter le roulement des lits et empêcher l'engorgement du système par des individus qui sont médicalement stables, même s'ils éprouvent encore des difficultés de fonctionnement dans la vie de tous les jours. Ce processus garantit la disponibilité de lits aux personnes qui nécessitent des soins aigus.

Peu avant mon entrée en fonction à l'hôpital, certaines personnes hospitalisées devaient attendre pendant plusieurs années qu'un lit se libère dans des établissements de soins de longue 
durée. Les ressources communautaires étant inadéquates pour répondre aux besoins de la communauté, le milieu hospitalier devait assumer la responsabilité de ce manque de services.

Aujourd'hui, la disponibilité des ressources, plus particulièrement les ressources financières, est encore très limitée. Ainsi, le milieu hospitalier n'est plus capable de pallier le manque de services communautaires. Notre clientèle francophone, en majorité économiquement défavorisée, doit souvent en subir les conséquences. Il est parfois difficile d'avoir accès à ces ressources pour les francophones. Par exemple, dans la région, seuls deux des dix-huit établissements de soins de longue durée sont désignés francophones. Les listes d'attentes peuvent varier entre six et vingt-quatre mois avant d'avoir accès au service désiré. Jusqu'à présent, je n'ai pas connu de clients francophones en attente de placement qui ont réussi à être transférés du premier coup dans l'un de ces établissements. Habituellement, ces clients doivent être placés dans un autre établissement de soins de longue durée, soit anglophone ou situé à l'extérieur de la ville, en attendant leur placement dans un établissement francophone. En tant que travailleuse sociale, j’ai la responsabilité d'aider les bénéficiaires et leur famille à trouver des options aux services qui ne leurs sont pas immédiatement disponibles. Malheureusement, la plupart du temps celles-ci sont très limitées et ne répondent pas aux désirs des clients, ni à ceux de leurs familles.

J'entends parfois certains professionnels du système de santé dire que les bénéficiaires dépendent inutilement de nos services pour répondre à leurs besoins sociaux. Parfois, ceux-ci se présentent à la salle d'urgence en espérant être admis afin d'échapper à leurs problèmes familiaux ou sociaux. Ces professionnels de la santé ne comprennent pas que l'intervention de type unilatéral, qui caractérise l'approche institutionnelle, alimente cette dépendance au système. Le système institutionnel perçoit les bénéficiaires comme des êtres passifs, incapables de se prendre en charge. L'approche institutionnelle favorise ainsi les inégalités entre l'aidant et l'aidé en excluant les bénéficiaires du processus de décisions concernant leur bien-être. À la longue, cette approche empêche l'échange réciproque de la personne avec son environnement et propage l'exclusion et la marginalisation sociale. 
Lors de mes interventions individuelles, je tente d'inclure l'individu dans le processus de définition et de résolution de ses problèmes afin de l'encourager à se prendre en main. Ces habiletés l'aideront lorsqu'il prendra congé de l'hôpital à développer et à maintenir son autonomie. Comme l'ont démontré les répondants dans mon mémoire, les personnes ayant des handicaps sont des personnes débrouillardes qui désirent avoir un certain contrôle sur l'aide qu'elles reçoivent des autres. Ce travail est un long processus qui exige beaucoup de temps et d'efforts de la part du client et de l'intervenant. Or, ce type d'intervention, dans un milieu institutionnel, est malheureusement perçu comme une perte de temps, augmentant inutilement la durée du séjour du client. Il arrive parfois que les désirs de la personne ne soient pas toujours conformes au plan de traitement proposé par les professionnels de la santé. À ce moment, j'assume le rôle de médiatrice afin de permettre aux deux parties d'arriver à un compromis, tout en respectant le besoin de l'usager face à son pouvoir décisionnel en vue de maintenir son autonomie.

\section{Le cas}

J'ai participé récemment à une conférence de cas dans la communauté où étaient présents le consommateur du système de la santé, les diverses ressources communautaires impliquées et des représentants de chaque hôpital de la région. Le consommateur étant bien connu de tous les hôpitaux, l'objectif de la rencontre était d'explorer les diverses options pour réduire la fréquence de ses visites aux salles d'urgence. Comme l'a mentionné une intervenante de la santé: "You're costing a lot of money to the system». Et tous les professionnels présents disaient que les problèmes sociaux du bénéficiaire seraient mieux contrôlés, si nous pouvions prévenir la dégénération de ses problèmes médicaux.

Cette personne ayant un handicap physique est sans abri et souffre d'un problème de toxicomanie de longue date. Son manque de ressources financières la pousse à consommer de l'alcool à friction pour satisfaire sa dépendance. Dans le passé, le client a participé, sans grand succès, à plusieurs programmes de 
réadaptation en toxicomanie. Il y a environ un an, il avait exprimé le désir de se trouver un logement stable et sécuritaire, car il ne voulait plus vivre dans les centres d'hébergement d'urgence. Une résidence supervisée située à l'extérieur de la ville, était prête à l'accueillir, à condition qu'il ne consomme plus. Finalement, il n'y est pas resté longtemps, car sa dépendance à l'alcool a repris le dessus. Plusieurs ressources communautaires étant épuisées, le client se retrouve avec très peu de soutien social.

Les gens présents à la conférence tentaient de trouver une solution pour l'aider à améliorer ses conditions de vie. Leur désir de prendre en charge la vie du bénéficiaire était frappant. Certains ont voulu qu'il soit admis dans un programme de désintoxication pour traiter son problème de toxicomanie. Malheureusement, le centre de désintoxication est incapable d'accommoder les personnes en perte d'autonomie physique. D'autres ont proposé de le faire admettre dans un hôpital psychiatrique afin d'évaluer sa compétence à prendre soin de lui-même. Ceux-ci pensaient que le Bureau du curateur public avait la responsabilité de ses soins, car ils prétendaient que le bénéficiaire prend de mauvaises décisions par rapport à son bien-être. Un médecin a finalement répondu que le patient a le droit de prendre des mauvaises décisions par rapport à son bien-être et qu'il était capable légalement de prendre des décisions par rapport à ses soins.

J'étais assise près du client lors de la rencontre. Il semblait intimidé par tous les professionnels assis autour de la table. Parfois, j'ai remarqué que des larmes coulaient sur ses joues. Il était évident que le sujet de la discussion le bouleversait. Il semblait coupable d'être incapable de répondre aux attentes des professionnels.

Après quarante minutes de discussion, je lui ai demandé s'il désirait obtenir de l'aide pour son problème de toxicomanie. La rencontre était jusque là orientée autour de notre perception de son bien-être. Il a répondu, «I would like to beat this addiction, but at this time, I couldn't care less». J'ai suggéré que nous tentions de l'aider à maintenir son état de santé et à prévenir une détérioration, tout en respectant ses choix. À partir de ce moment, la discussion a radicalement changé. Le centre d'hébergement d'urgence a dit qu'il tenterait de satisfaire son besoin de 
consommation en lui fournissant une boisson alcoolisée qui serait moins nuisible que l'alcool à friction. Le centre a aussi accepté de l'héberger le jour et la nuit afin d'assurer sa sécurité. Finalement, je me suis engagée à lui trouver un intervenant de rue, prêt à l'aider à maintenir un niveau de bien-être satisfaisant. À cette étape du processus, le client considérait prématuré d'évaluer la pertinence des interventions prises à son sujet.

Les professionnels de la santé ont tendance à croire qu'ils possèdent les connaissances nécessaires pour régler tous les problèmes des bénéficiaires. On oublie parfois que les consommateurs de services possèdent les habiletés nécessaires pour se prendre en charge et que notre rôle ne consiste qu'à faciliter leur trajet.

\section{Conclusion}

Les personnes répondantes de mon mémoire ont maintes fois souligné l'importance qu'elles attribuent à la liberté de prendre des décisions concernant leur bien-être et d'interagir activement avec les gens de leur entourage. L'intervention professionnelle, par contre, a tendance à prendre en charge le traitement des bénéficiaires, en présumant connaitre ce qui est le mieux pour eux. Contrairement aux principes de l'intervention favorisée par le système institutionnel, les répondants ont démontré qu'ils sont des personnes actives, capables d'assumer la responsabilité de leur propre vie, en développant quotidiennement des stratégies pour surmonter les obstacles, l'autonomie étant l'une des valeurs qui dominent leurs actions.

Le système de la santé subit beaucoup de coupures budgétaires. Les changements structuraux dans le système médical feront en sorte que l'approche institutionnelle ne pourra plus se permettre de prendre en charge la vie des clients. Les hôpitaux doivent, de plus en plus, dépendre des organismes communautaires et des unités familiales pour assurer la continuité des soins, malgré 
l'effondrement actuel du tissu social. Malheureusement, l'approche institutionnelle ne semble pas évoluer à la même vitesse que les changements qui lui sont imposés. Le milieu institutionnel semble être mené par ses contraintes budgétaires. En effet, depuis quelques années, le système de la santé subit des pressions économiques accrues qui l'oblige à se préoccuper davantage du coût des services offerts que de leur impact sur la qualité de la vie humaine. 